\title{
Broadening Our \\ Understanding of Predictors of Subjective Age
}

\author{
Gretchen A. Petery, M.A. \\ Janet Barnes-Farrell, Ph.D. \\ Rick A. Laguerre, B.A. \\ Jacklyn Scymcyk, B.A.
}

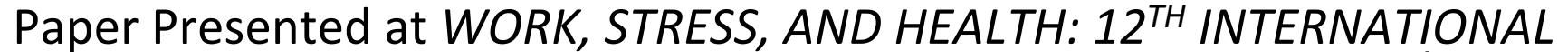
CONFERENCE ON OCCUPATIONAL STRESS AND HEALTH, Minneapolis, MN, June 2017

This work was supported by Grant Number T01-OH08610 from the National Institute for Occupational Safety and Health. Its contents are solely the responsibility of the authors and do not necessarily represent the official views of NIOSH. 


\section{Subjective Age: An Alternative to Chronological Age}

Measurement

- Multi-faceted

- "How old do you feel?" (Felt age)

Better predictor than chronological age
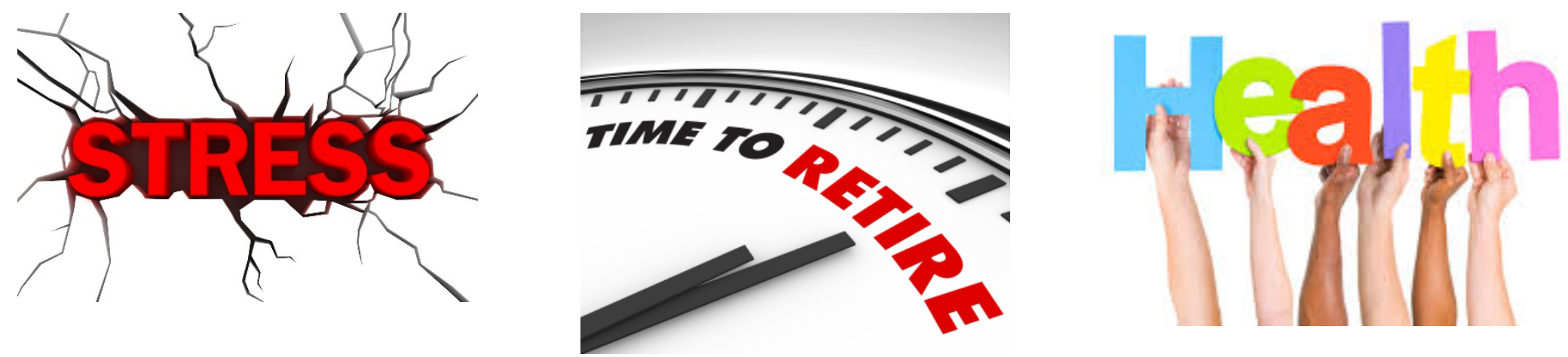


\section{Predictors of Subjective Age?}

- Mostly from marketing and gerontological research

- Theory driven focus for selection

$>$ May hinder progress when phenomenon is not well understood

- Why not simple ask why?

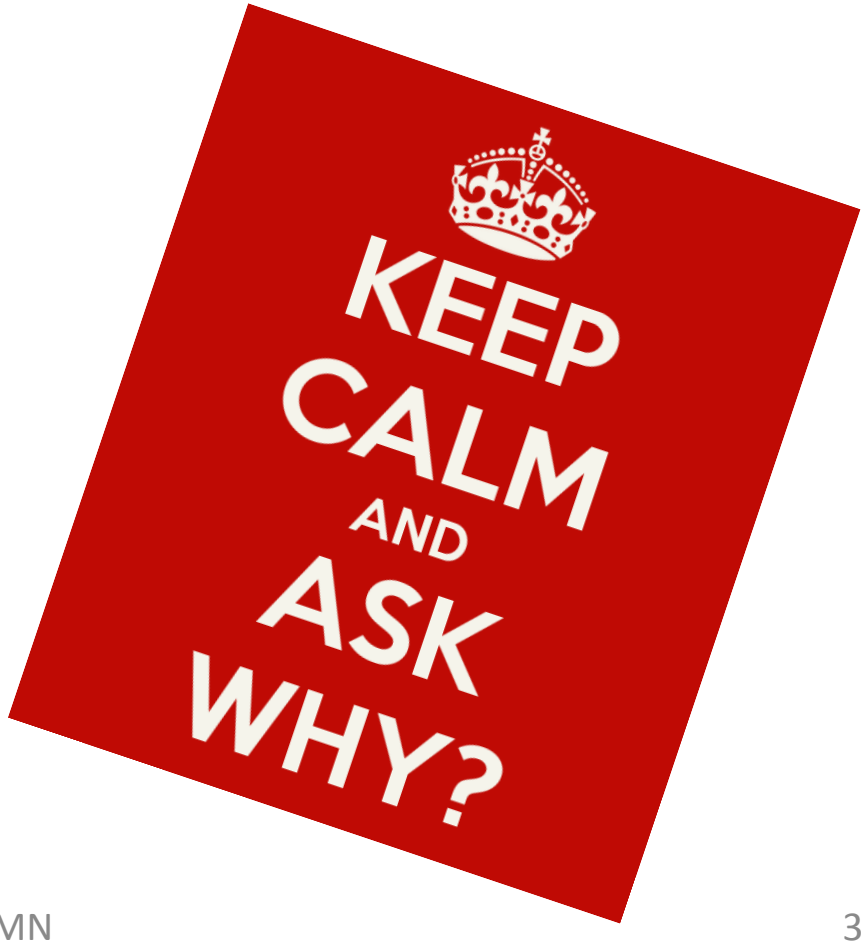




\section{Methods}

417 individuals surveyed

- Felt Age

$>$ Discrepancy calculated: FA - CA

$\checkmark$ Negative value $=$ feeling $X$ years younger

$\checkmark$ Positive value $=$ feeling $X$ years older

- "Why do you feel older or younger than your chronological age?"

224 (53.7\%) provided CA, FA, and usable reason

$>$ Male: $n=82$ (36.6\%)

$>$ Female: $n=141(62.9 \%)$

$>$ Age: $M=35.3$ (16.1), range 18-74

$\checkmark$ Grouped in CA categories: Young, Middle-age, "Old" 


\section{Sample size $=224$}

Percentage by Age Categories

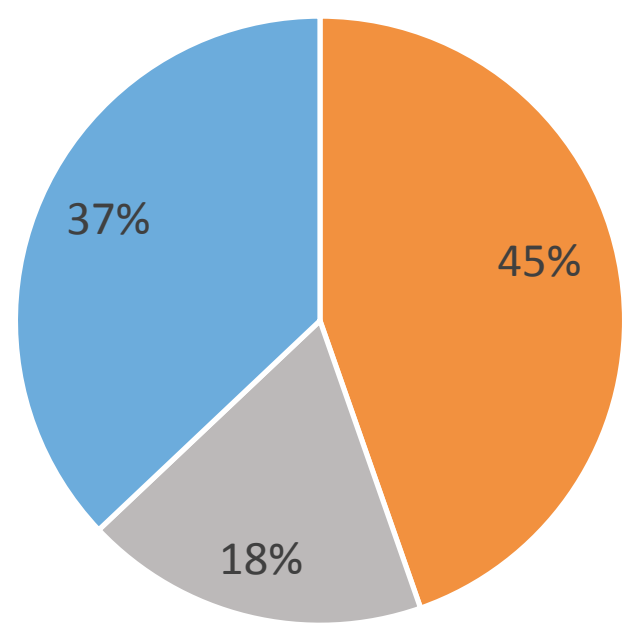

- Young" 18-24 yrs old $(n=100)$

- Middle Age: 25-44 yrs old $(n=41)$

- "Old": 45 and older $(n=83)$

\section{Percentage by Felt Age}

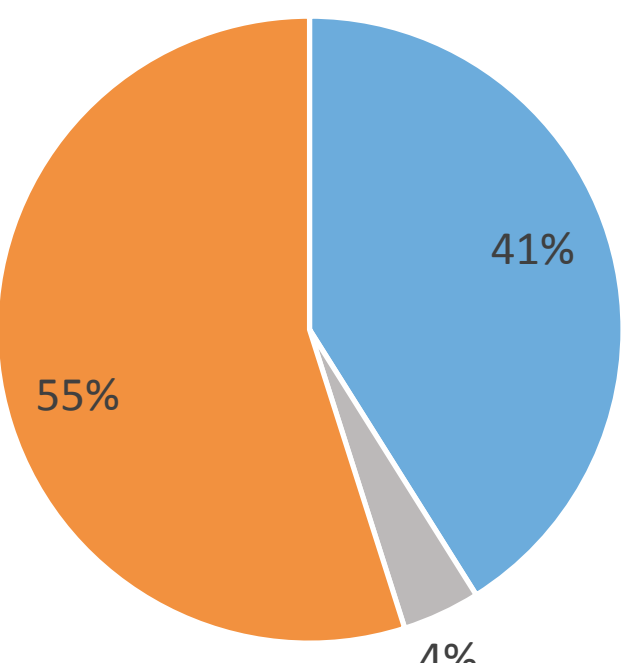

$4 \%$
- Older than CA ( $\mathrm{n}=92)$

- Same as CA ( $n=9)$

- Younger than CA $(n=123)$ 


\section{Procedure}

\section{Independent}

Coding of Themes

- Multiple themes possible per response

- 50 unique themes emerged

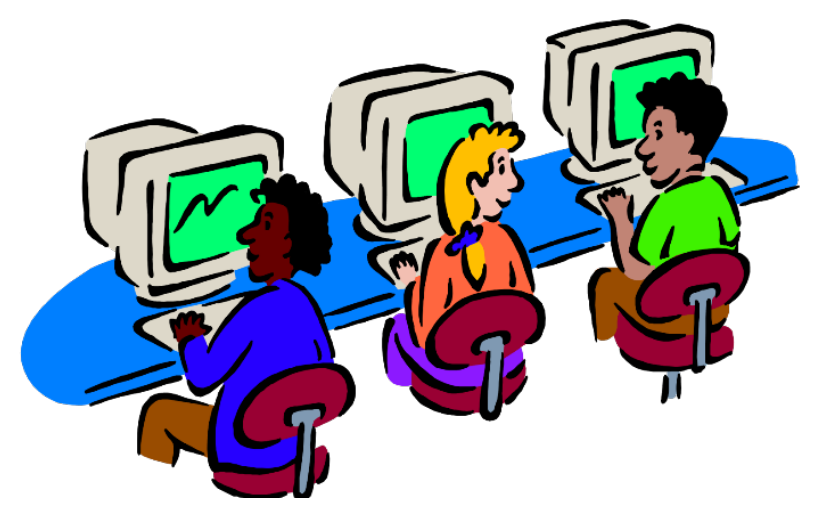

UCDNN

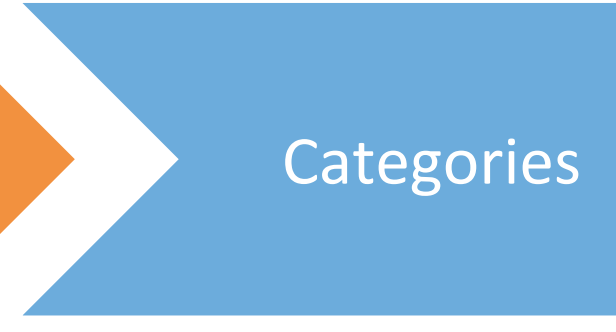

- First author sorted themes into broad categories
Independent sorting of themes into categories

- Rater disagreement discussed until consensus reached
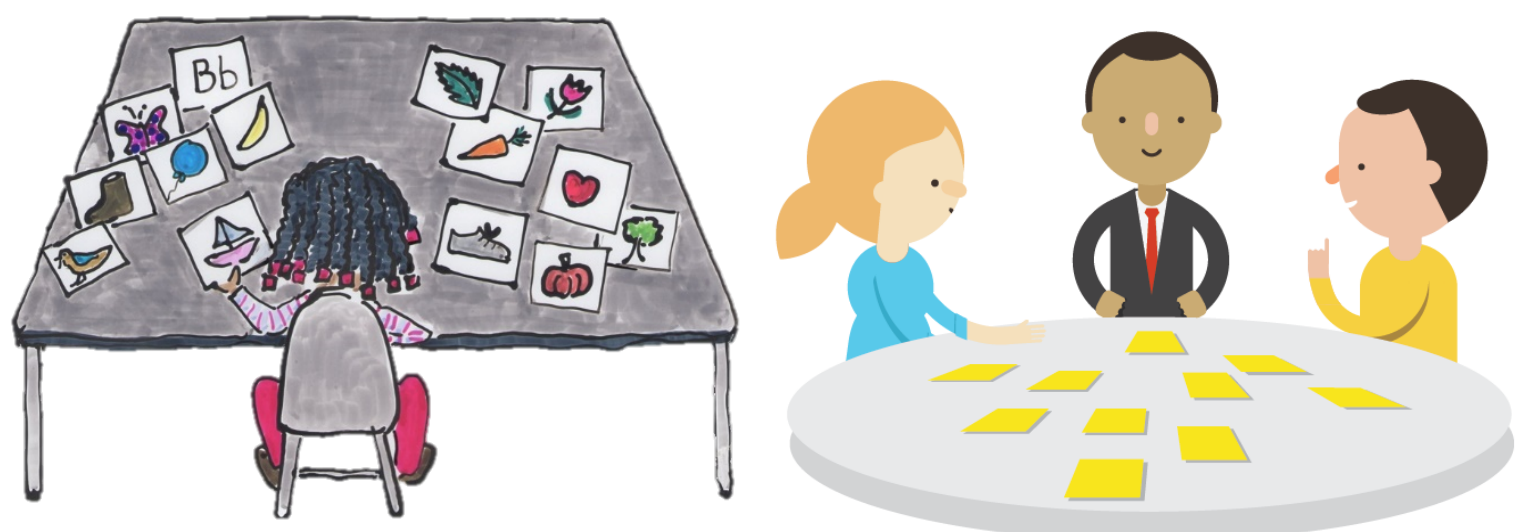


\section{Nine Categories (\% of responses)}

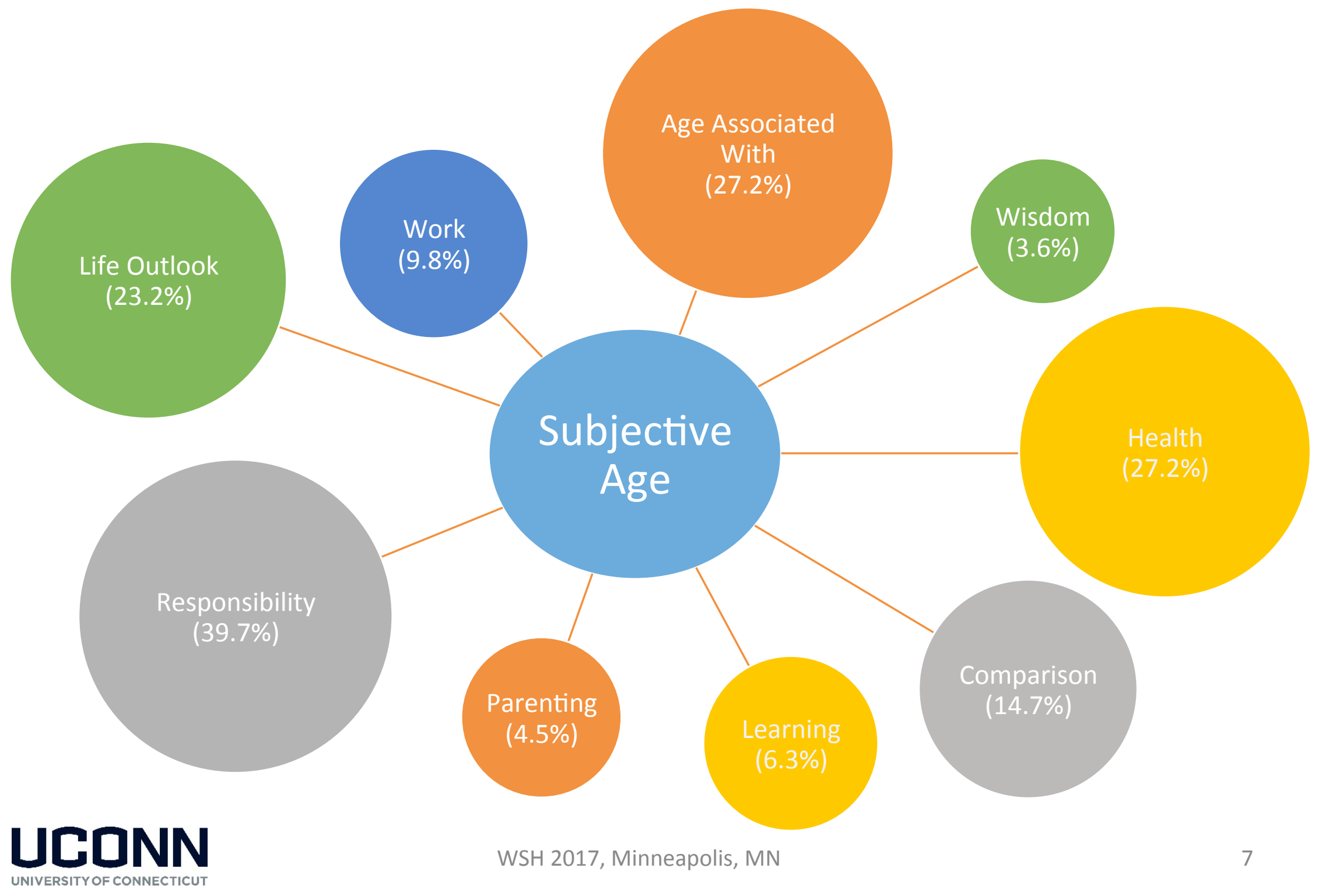




\section{Example Statements by Category}

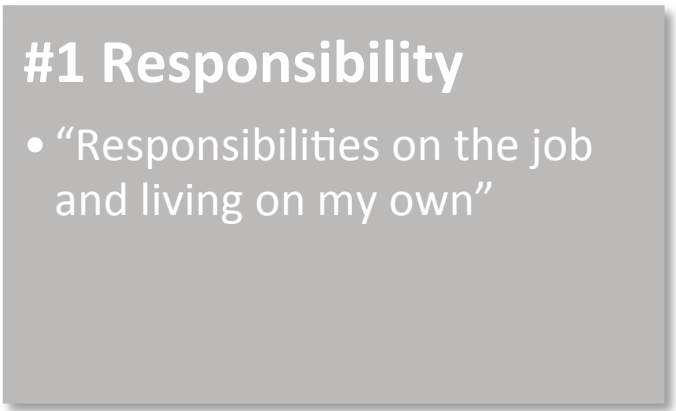

\section{\#4 Life Outlook}

- "I feel younger because I have a positive outlook on life and live life to the fullest"

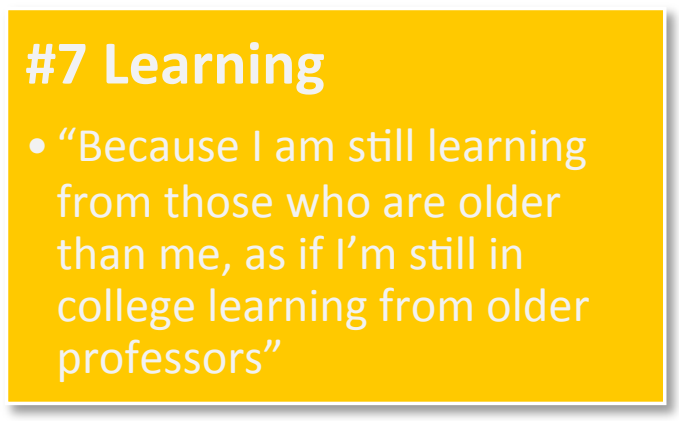

\section{\#2 Health}

- "Daily exercise and healthy eating"

\section{\#5 Comparison}

- "Feel I do not seem as old as the people around me who are similarly aged"

\section{\#8 Parenting \\ - "I am a single mom"}

\#3 Age Associate With

- "I feel like my interests are more similar to people a few years younger than I am than to people my age"

\#6 Work

• "Actively engaged in my job"

\section{\#9 Wisdom}

- "I have some wisdom" 


\section{Additional Research Questions}

- Are there categories that tend to co-occur?

- Are some categories more popular with different age groups or by gender?

- Were categories associated with feeling older or younger? 


\section{RQ1: Are there categories that tend to co-occur?}




\section{Number of Co-Occurrences of Categories}

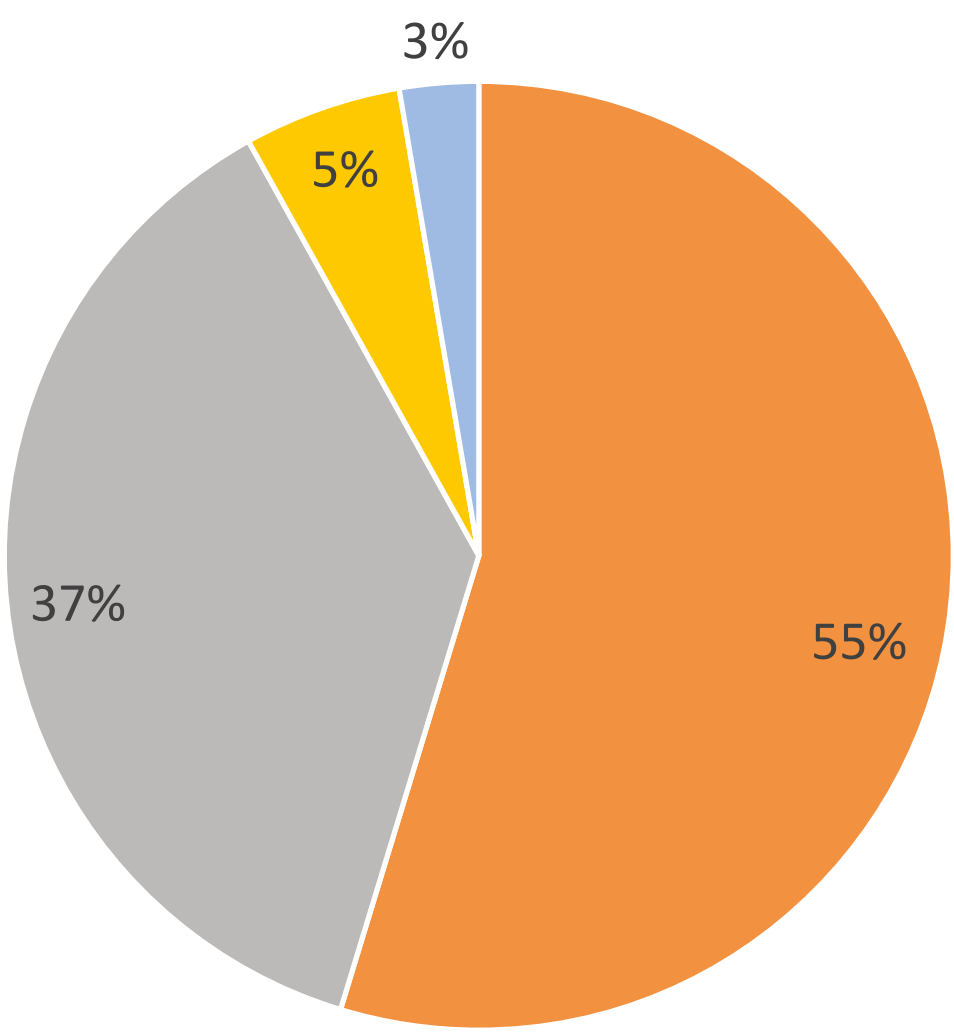

Overall

- 1 category

2 categories

3 categories

4 categories 


\section{Top Co-Occurring Categories}

\begin{tabular}{|c|l|}
\hline Responsibility & • Comparison (29\%) \\
\hline Health & - Life Outlook (24.6\%) \\
\hline Age Associate With & • Health \& Comparison (15.8\% ea) \\
\hline Life Outlook & • Health (30.2\%) \\
\hline Comparison & - Responsibility (72.7\%) \\
\hline Work & - Responsibility (52.4\%) \\
\hline Learning & - Health (50\%) \\
\hline Parenting & - Responsibility (50\%) \\
\hline Wisdom & - Responsibility (50\%) \\
\hline
\end{tabular}




\section{RQ2: Are some categories}

more popular with different age groups or by gender?

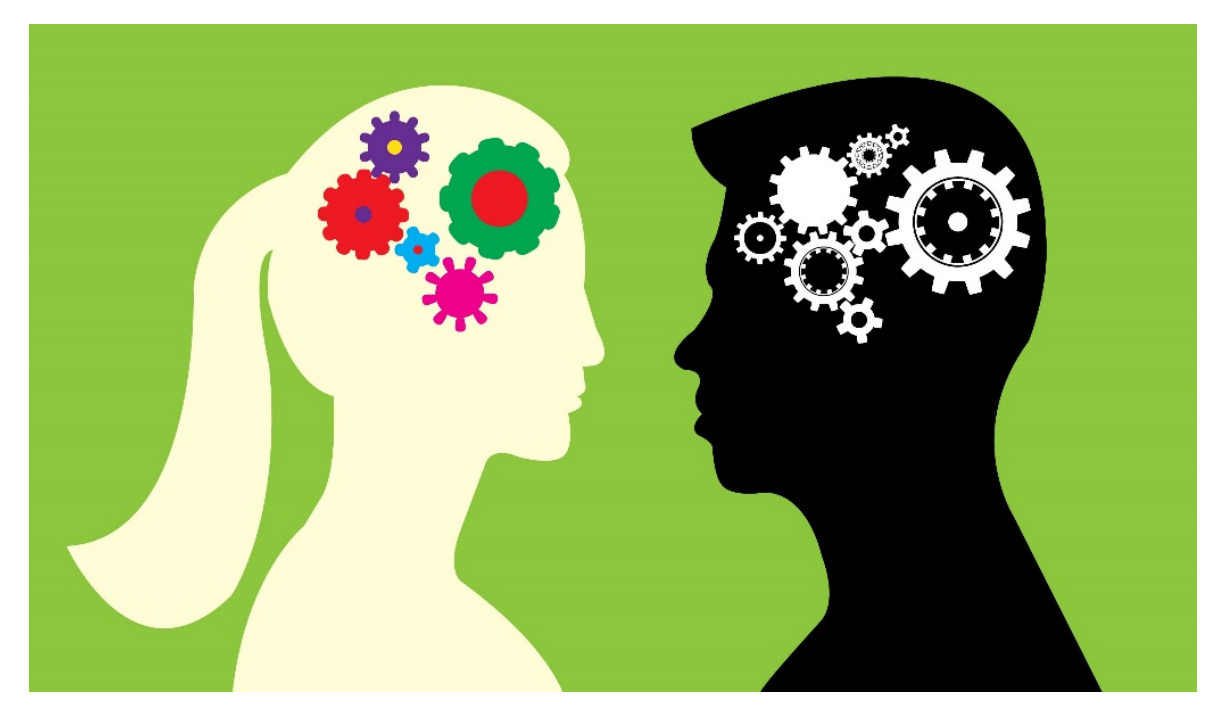




\section{Which age group used each category (\%)?}

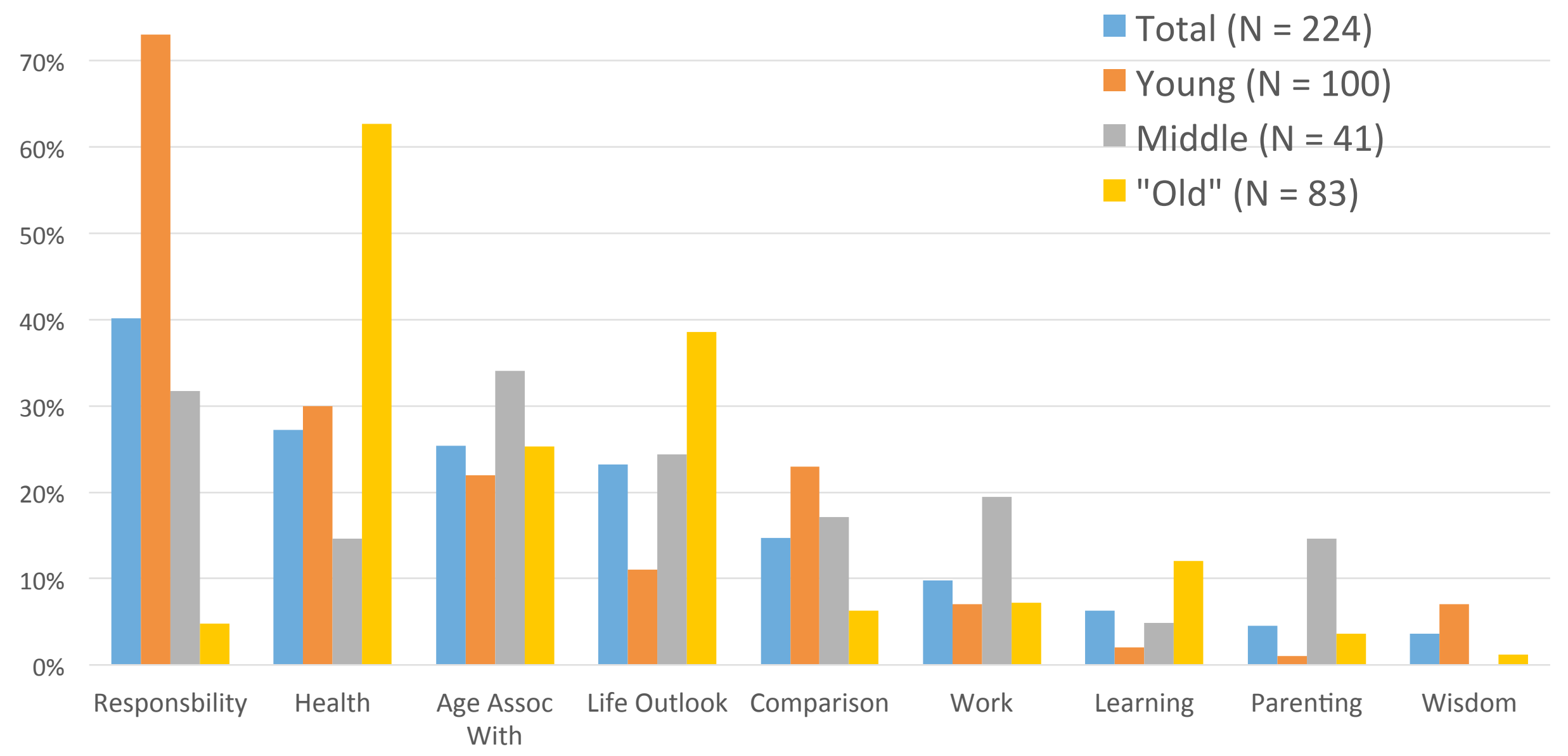




\section{Which age group used each category (\%)?}

$80 \%$

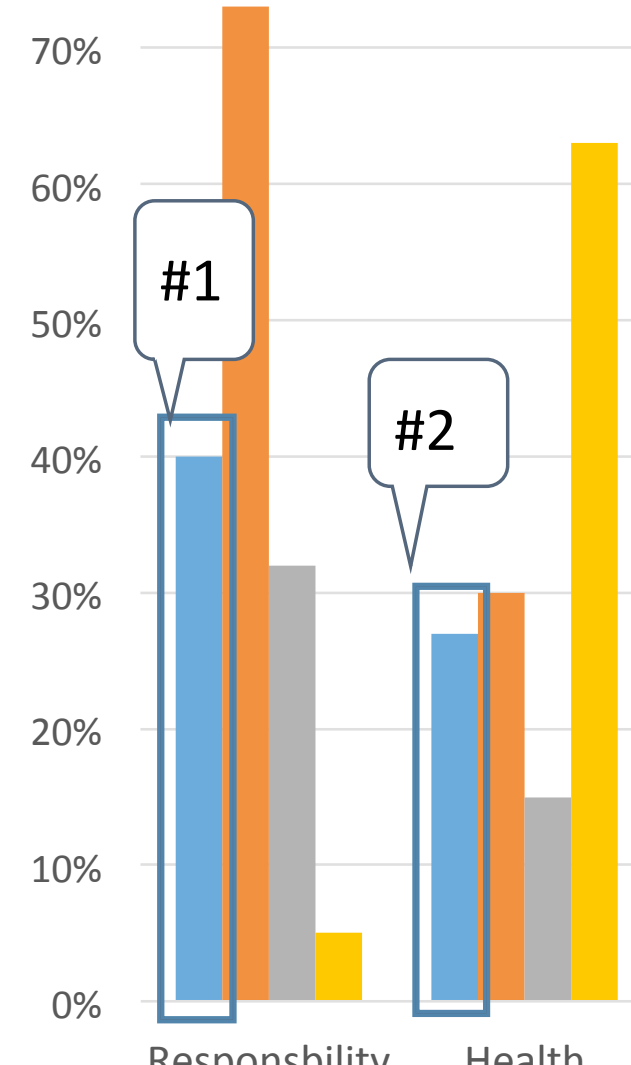

Responsbility Health

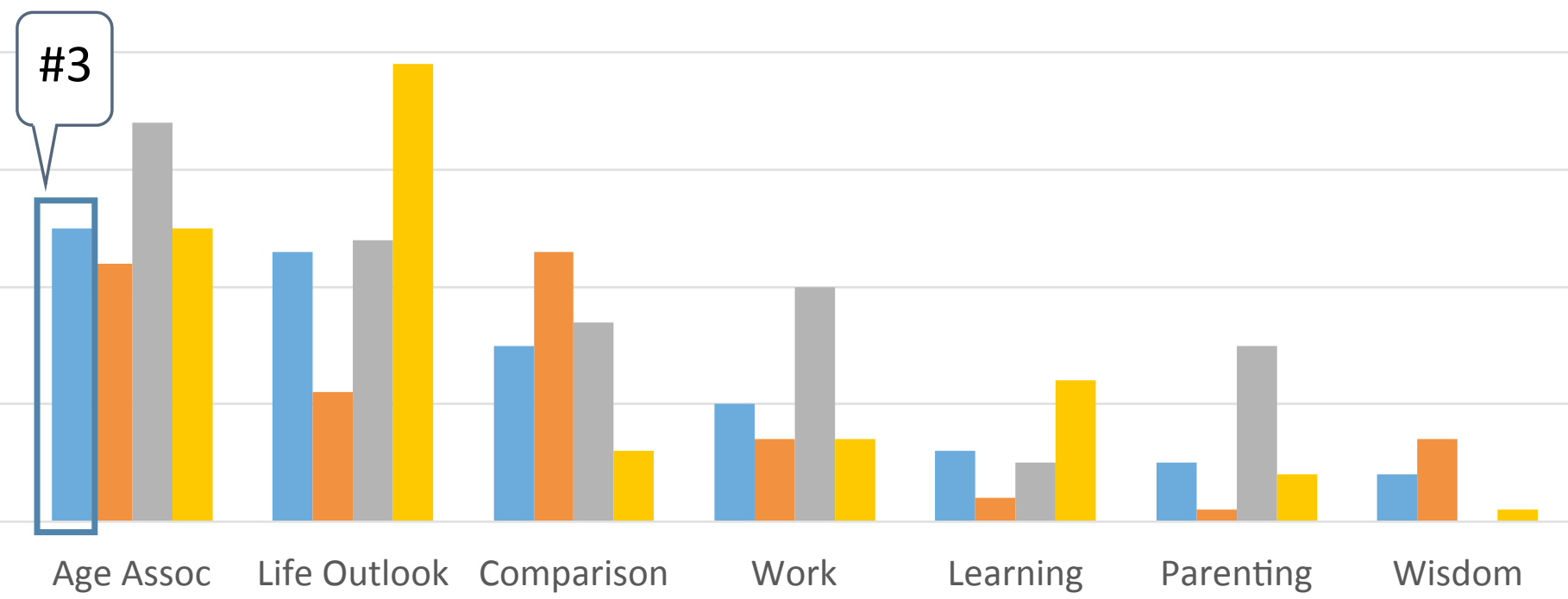

With

Total $(\mathrm{N}=224)$

Young $(\mathrm{N}=100)$

Middle $(\mathrm{N}=41)$

"Old" ( $\mathrm{N}=83)$ 


\section{Which age group used each category (\%)?}

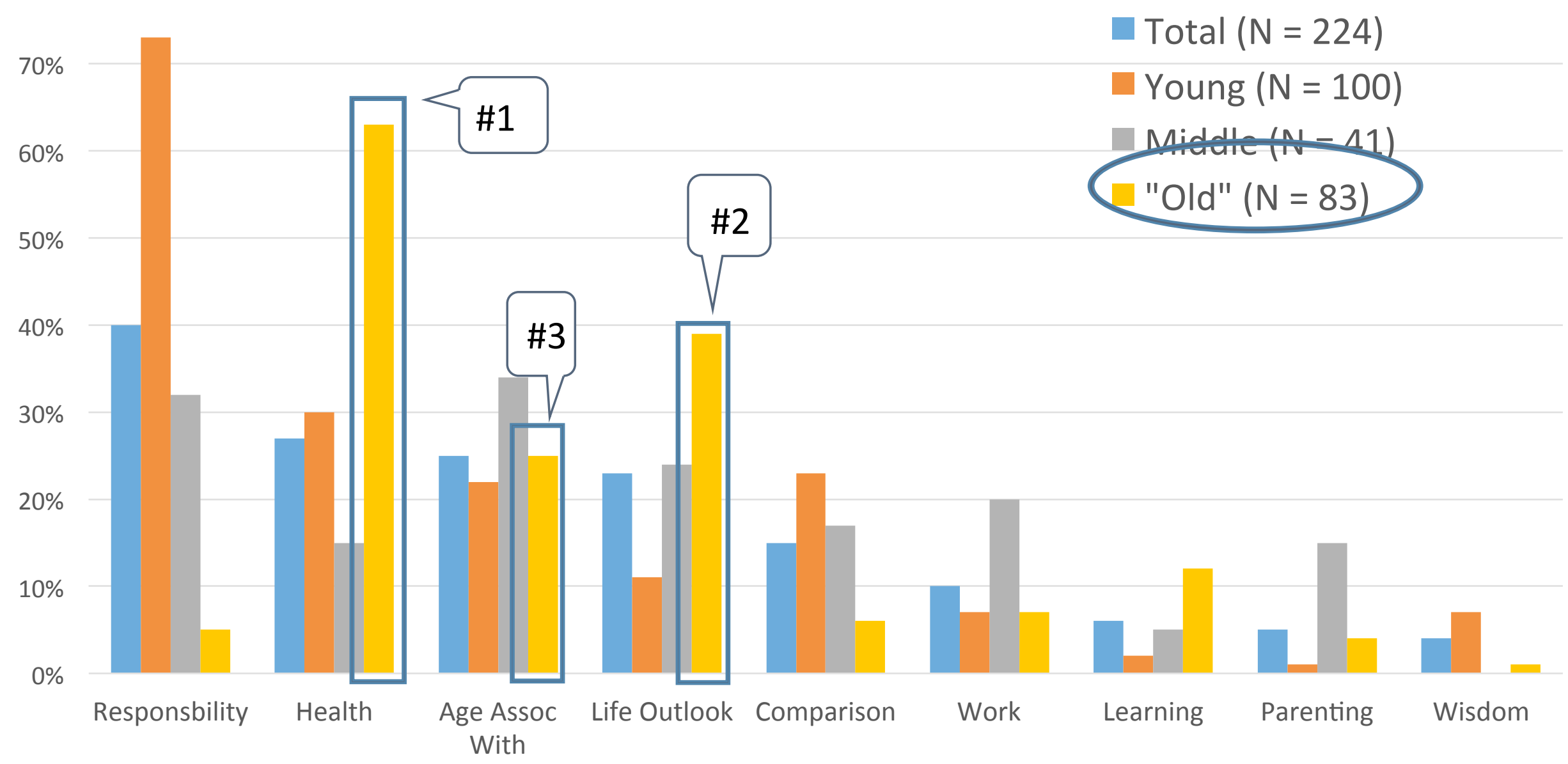




\section{Were there gender differences in the categories used (\%)?}

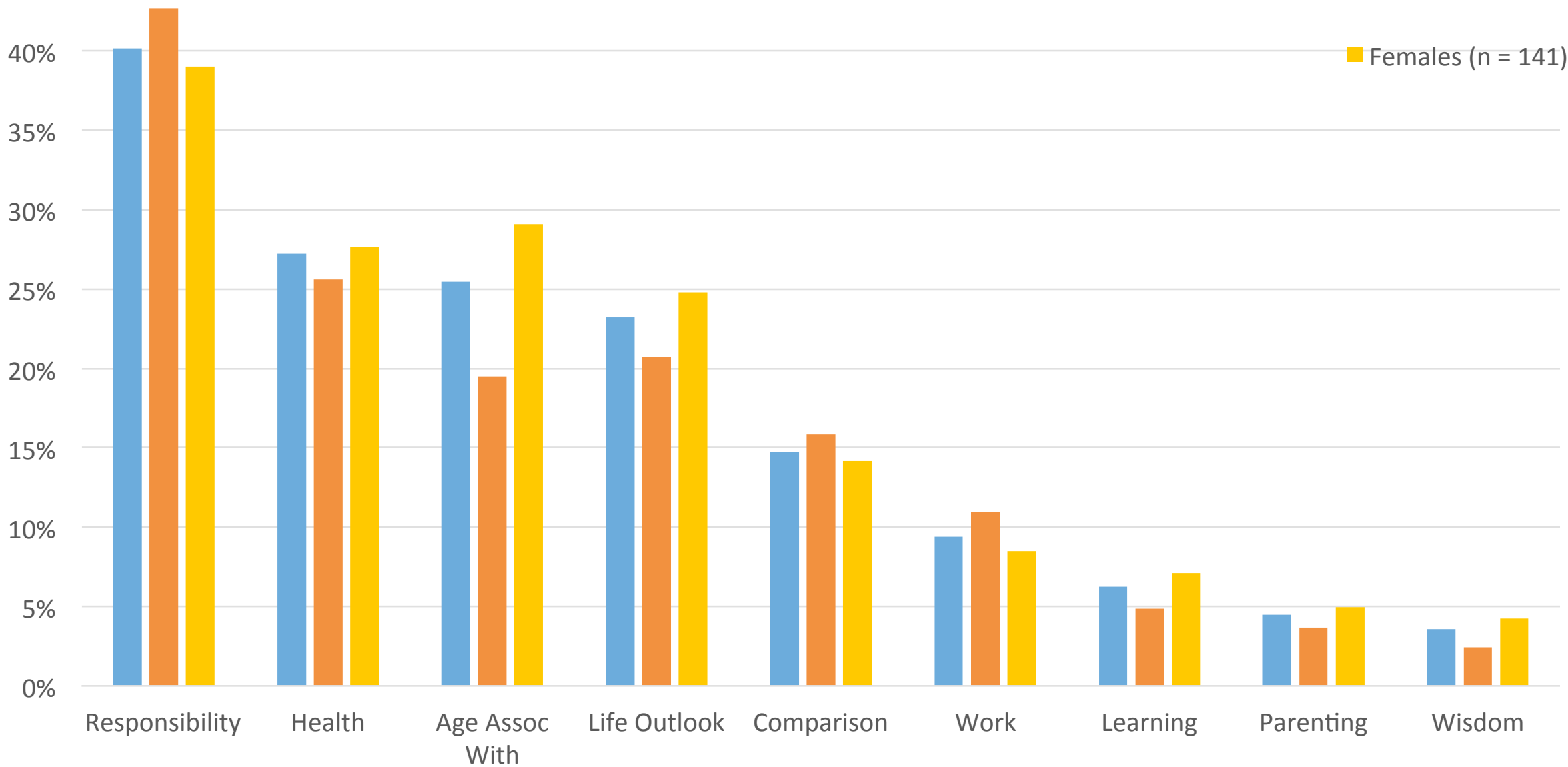




\section{Were there gender differences in the categories used (\%)?}

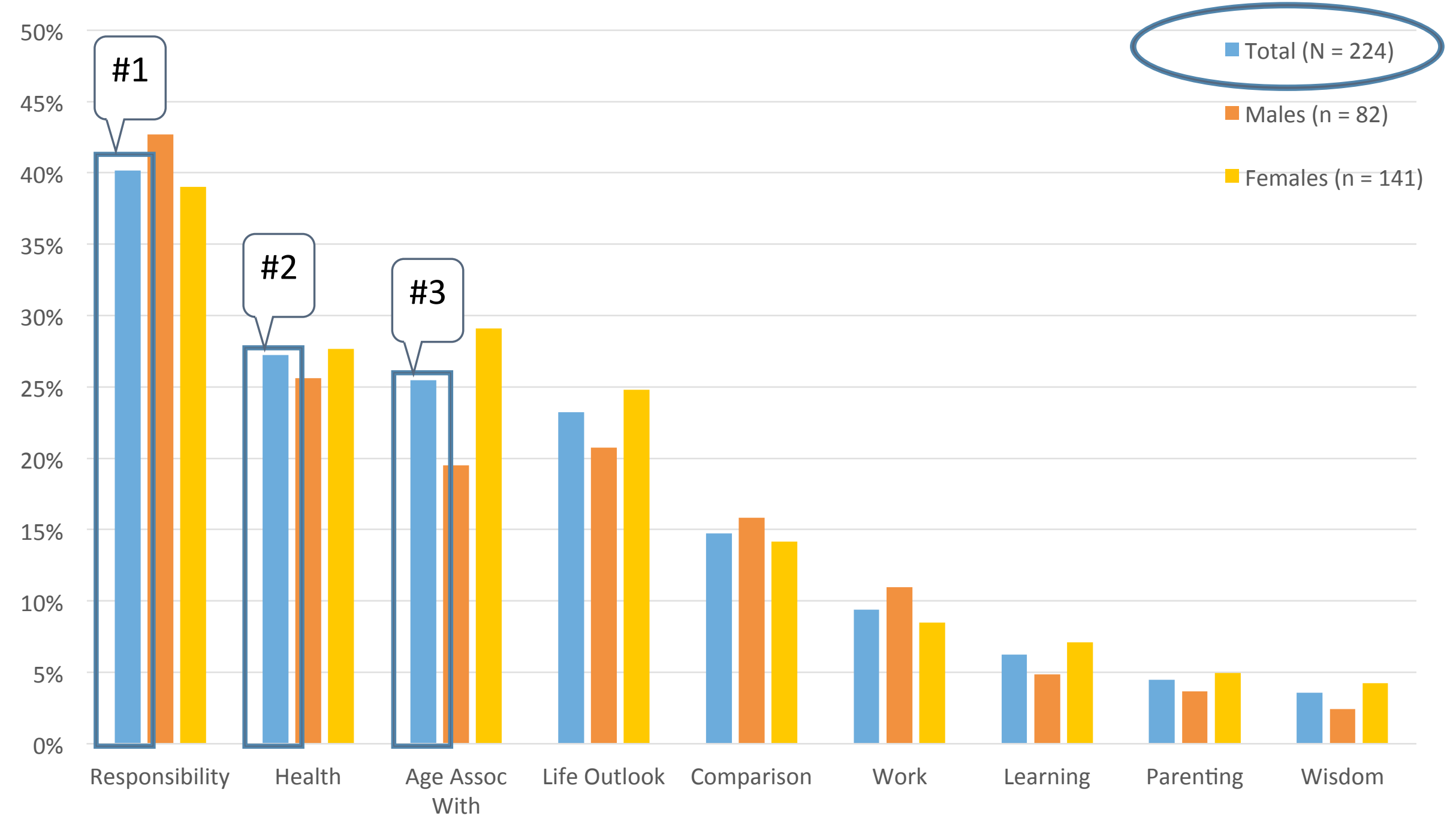




\section{Were there gender differences in the categories used (\%)?}

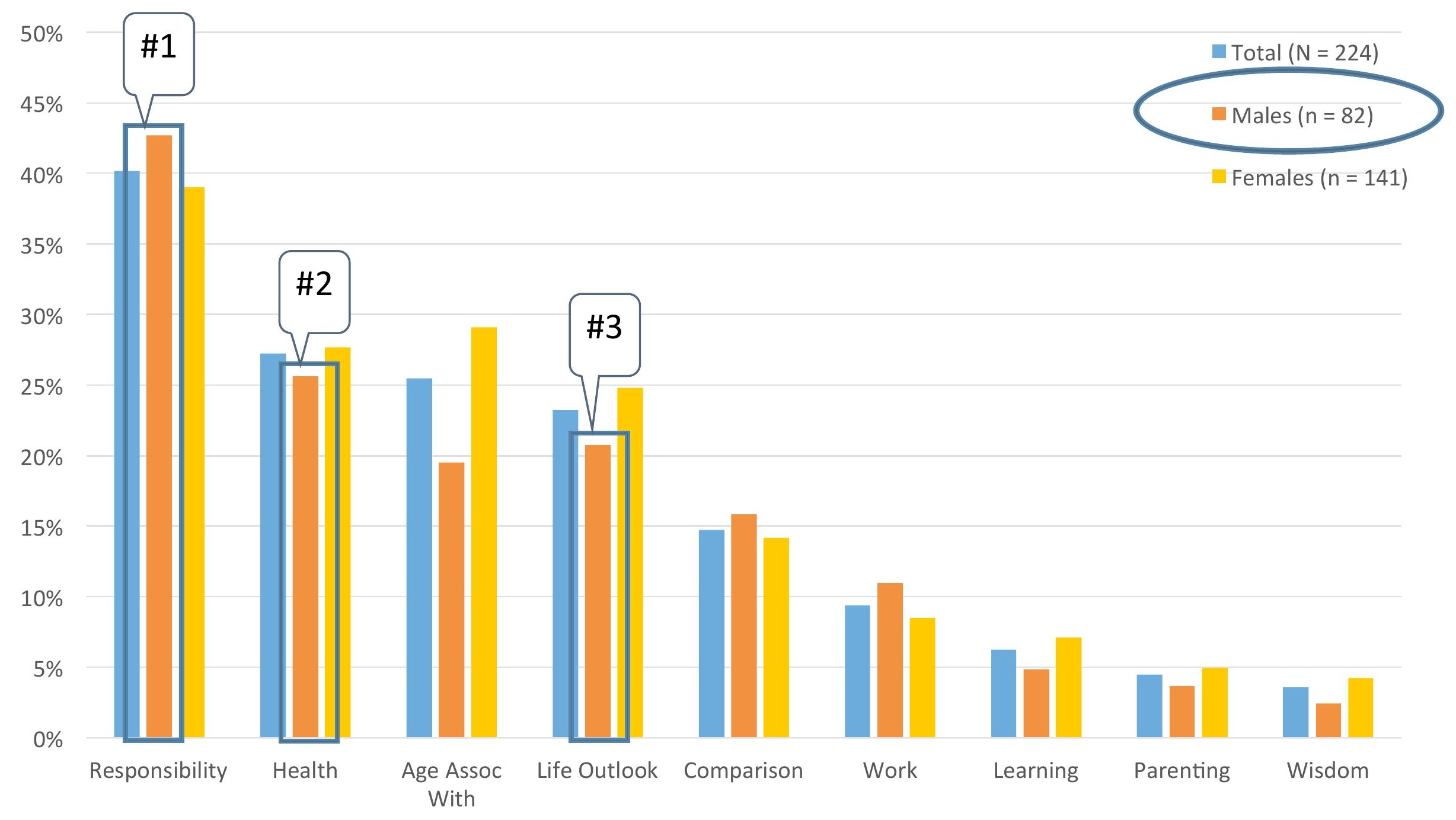




\section{Were there gender differences in the categories used (\%)?}

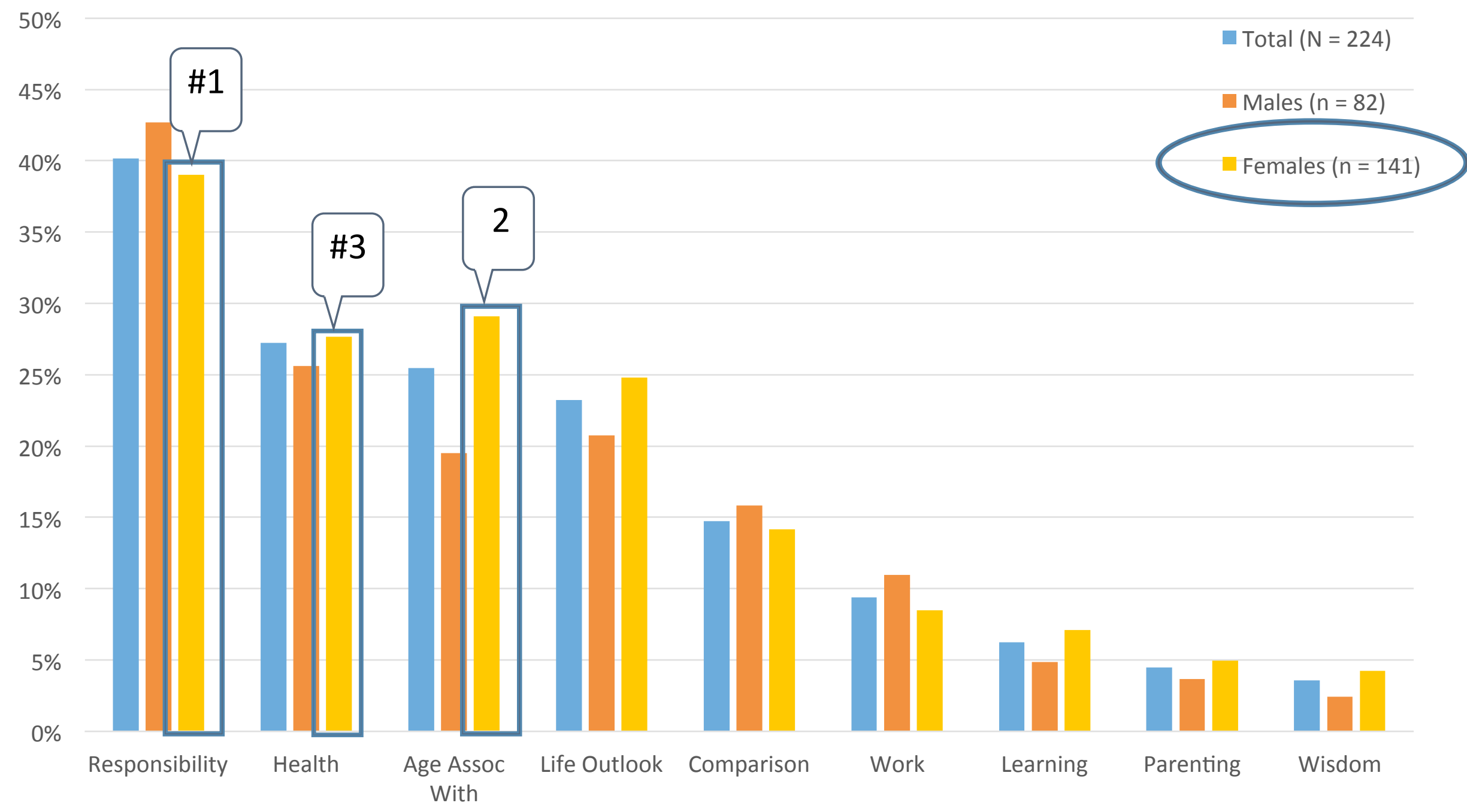




\section{RQ3: Were categories associated with feeling older or younger?}




\section{Feel Age Direction by Category}

Responsibility ( $N=90)$

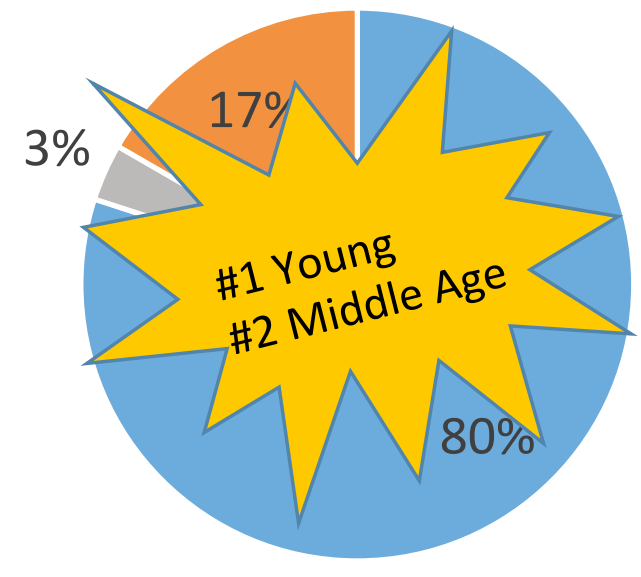

Health $(N=61)$

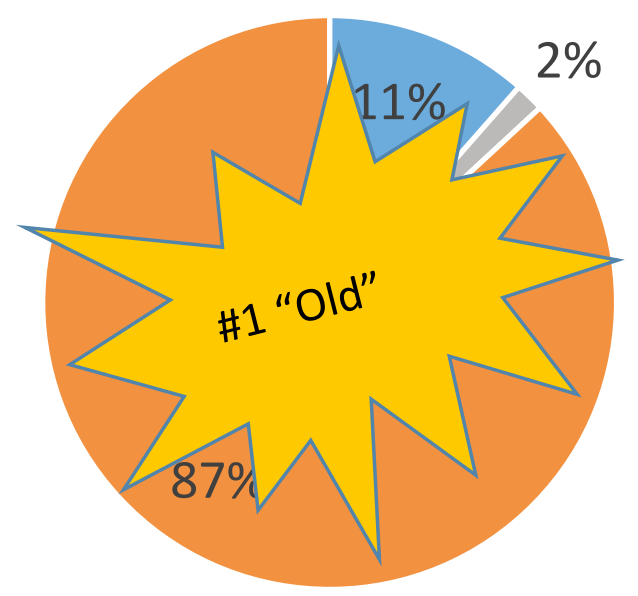

- Feel Older

- Feel Same

- Feel Younger
Age Assoc With ( $N=57)$

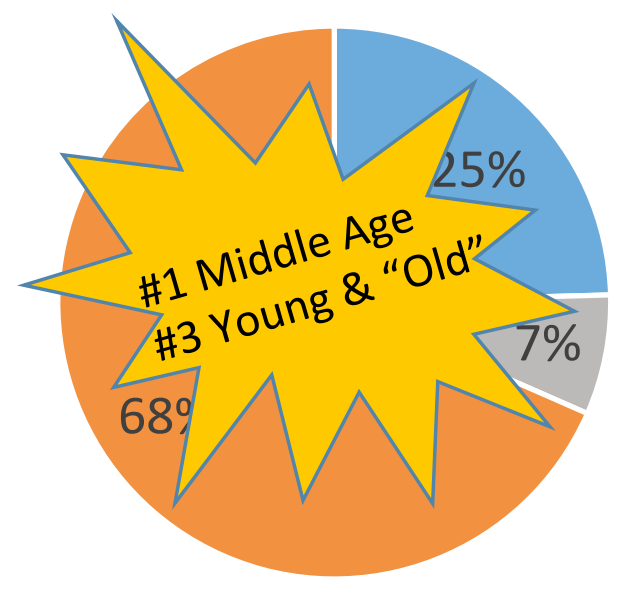




\section{Feel Age Direction by Category}

Life Outlook ( $N=52)$

Comparison $(\mathrm{N}=33)$

Work $(N=21)$
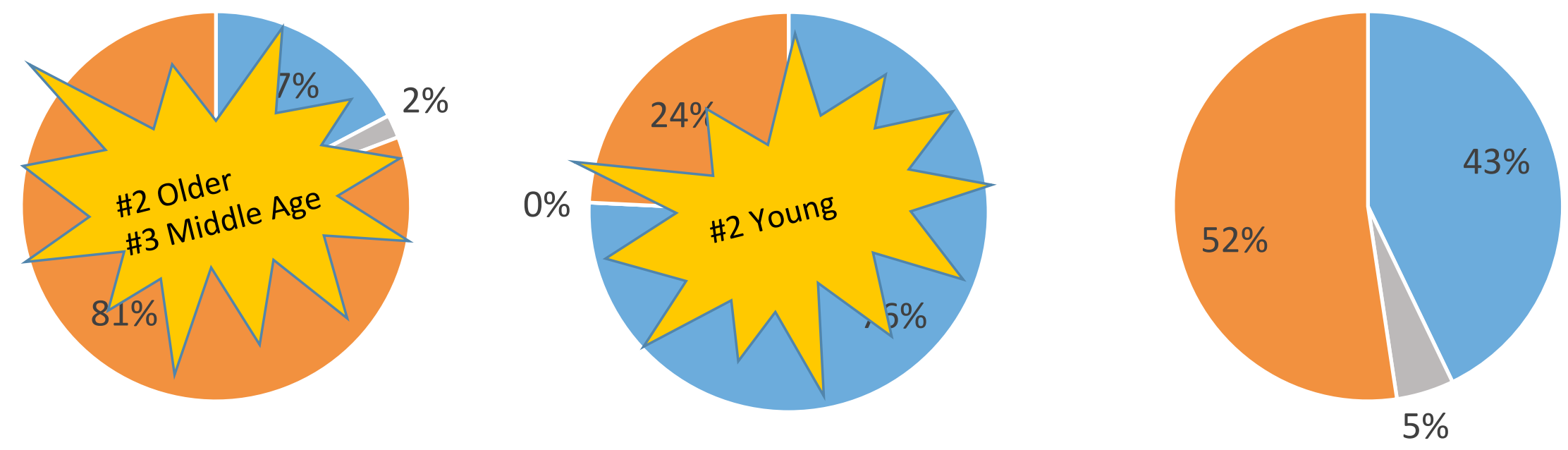

- Feel Older

- Feel Same

- Feel Younger 


\section{Feel Age Direction by Category}

Learning $(N=14)$

Parenting $(\mathrm{N}=10)$

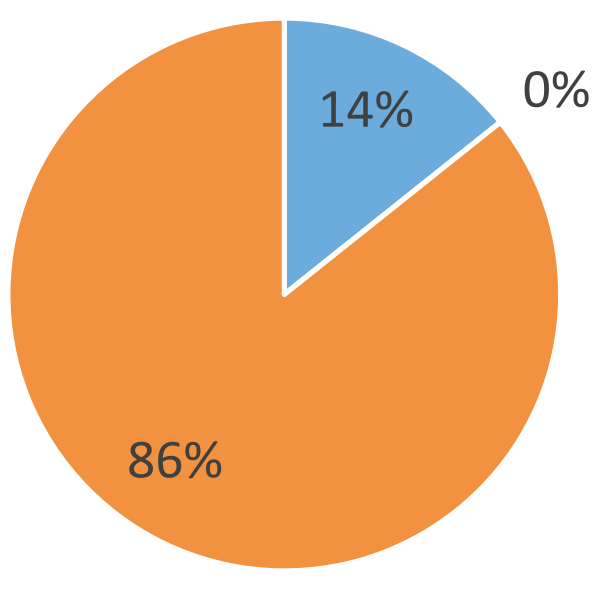

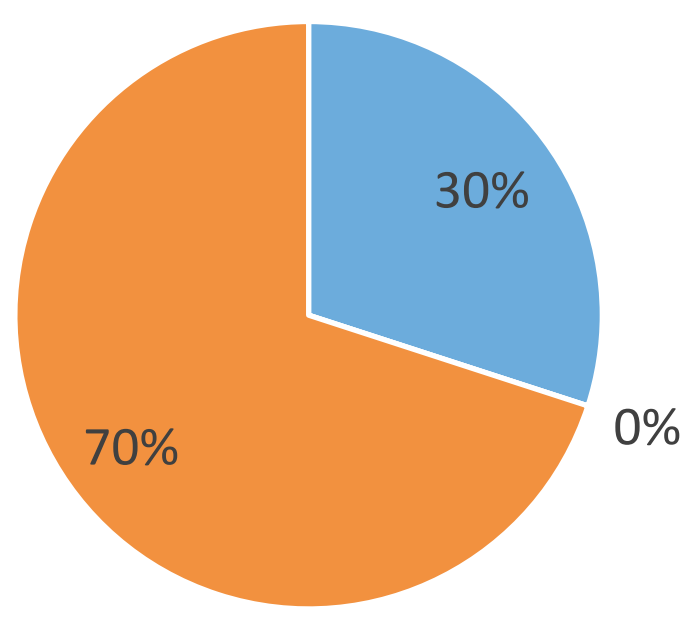

- Feel Older

- Feel Same

- Feel Younger
Wisdom $(\mathrm{N}=8)$

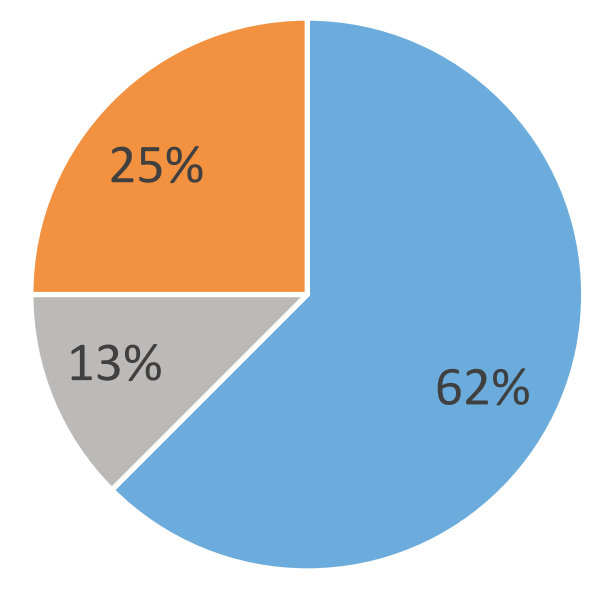




\section{Summary}

- Qualitative study, Descriptive results

- Additional predictors of subjective age identified

- Nine, non-exclusive categories

$>$ Multiple reasons why

$>$ Different categories for age groups and gender

- Categories and direction of felt age

$>$ Differences related to chronological age

- Counter intuitive findings

- Limitations

$>$ Skewed age and gender distribution

$>$ Convenience sample

- Next step

$>$ Replication and expansion

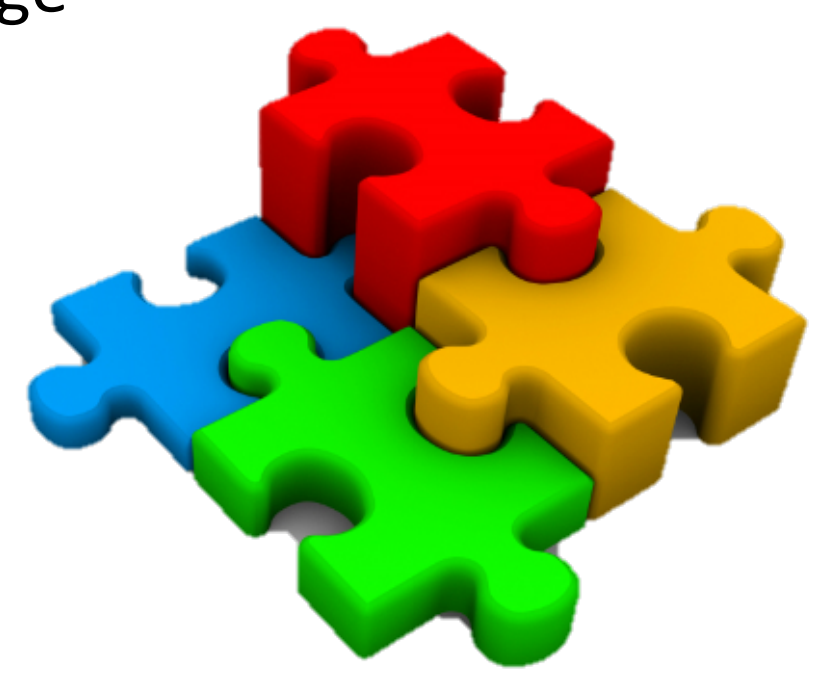




\section{Thank you!}

\section{Questions?}
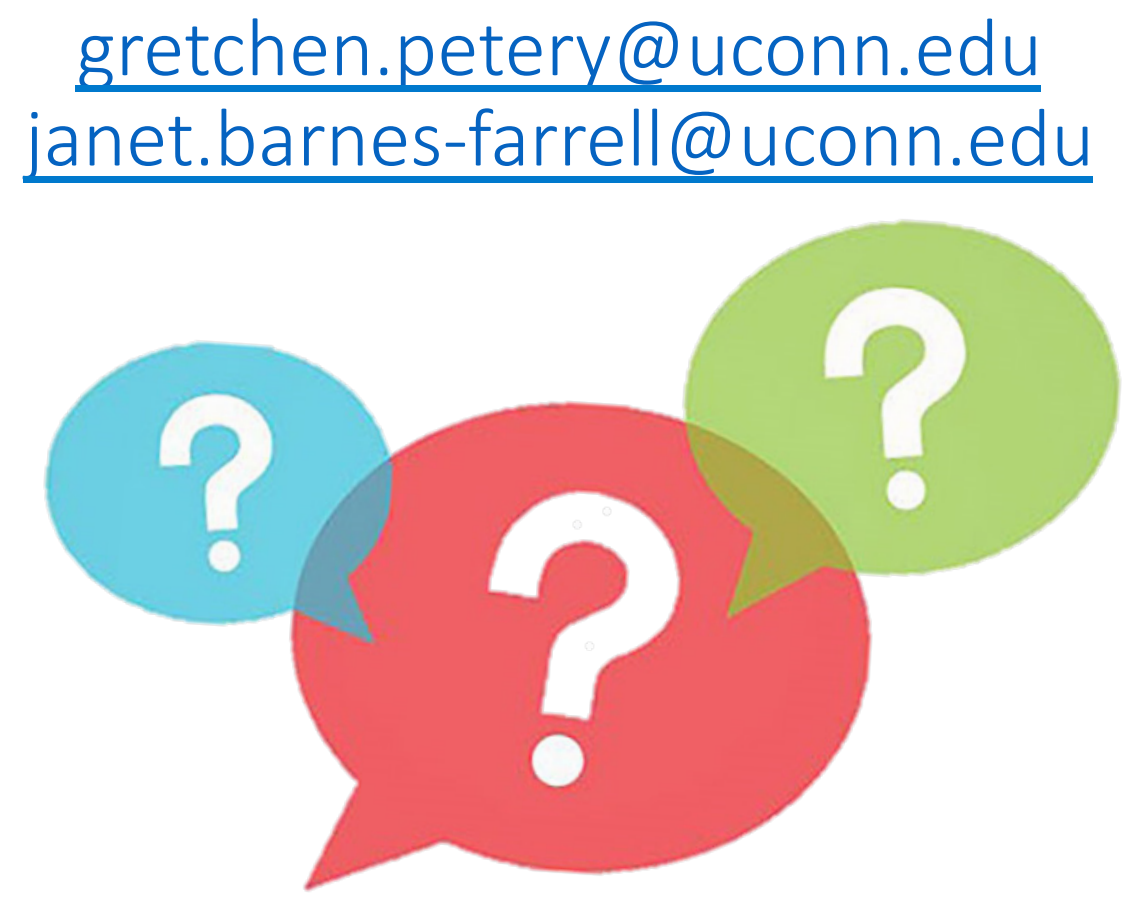
Supplemental Slides 


\section{Themes Within Categories}

\begin{tabular}{|c|c|c|c|c|c|}
\hline Responsibility & \multicolumn{2}{|c|}{ Health } & \multicolumn{2}{|c|}{ Age Associate With } & Life Outlook \\
\hline $\begin{array}{l}\text { - Responsibilities } \\
\text { - Maturity } \\
\text { - Experience } \\
\text { - Stress } \\
\text { - Burnout } \\
\text { - Focus } \\
\text { - Student } \\
\text { - Priorities }\end{array}$ & \multicolumn{2}{|c|}{$\begin{array}{l}\text { - Physical health } \\
\text { - Functional Health } \\
\text { - Health } \\
\text { - Aches \& pains } \\
\text { - Activity level } \\
\text { - Capabilities } \\
\text { - Diet } \\
\text { - Energy level } \\
\text { - Exercise/Workout }\end{array}$} & \multicolumn{2}{|c|}{$\begin{array}{l}\text { - Act age } \\
\text { - Unexpected feeling } \\
\text { - Interests } \\
\text { - Looks } \\
\text { - Trendy } \\
\text { - Unconventional life } \\
\text { - Views } \\
\text { - Treatment by others } \\
\text { - Interact with } \\
\text { different ages }\end{array}$} & $\begin{array}{l}\text { - Outlook } \\
\text { - Attitude } \\
\text { - Easy going } \\
\text { - Enjoyment } \\
\text { - Living full life } \\
\text { - Passage of time } \\
\text { - Social life } \\
\text { - Wishful thinking }\end{array}$ \\
\hline Comparison & Work & \multicolumn{2}{|c|}{ Learning } & Parenting & Wisdom \\
\hline - Comparison & - Work/Career & \multicolumn{2}{|c|}{$\begin{array}{l}\text { - Learning \& } \\
\text { development } \\
\text { - Mental } \\
\text { stimulation } \\
\text { - Technology }\end{array}$} & - Parenting & $\begin{array}{l}\text { - Wisdom } \\
\text { - Decisions }\end{array}$ \\
\hline
\end{tabular}




\section{Example Statements by Category}

Responsibility
- Young: "Responsibilities on
the job and living on my
own"
- Middle Age: "I feel about
my age because I am
beginning to feel burnout
and less motivated to
achieve more in my field
- "Old": "Tired of working"

\section{Responsibility}

Young: "Responsibilities on the job and living on my

Middle Age: "I feel about my age because I am and less motivated to

"Old": "Tired of working"

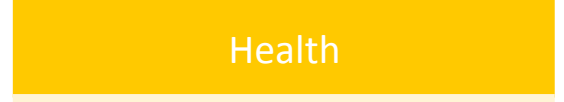

- Young: "Body pains"

- Middle Age: "Activity level"

- "Old": "Daily exercise and healthy eating"

\section{Age Associate With}

- Young: "Because time goes by too fast so I don't feel as old as I am"

- Middle Age: "I am surrounded by young people"

- "Old": "I feel like my interests are more similar to people a few years younger than I am than to people my age"

\section{Life Outlook}

- Young: "Starting to get a little jaded. Starting to learn what does and does not work for me"

- Middle Age: "I feel younger because I have a positive outlook on life and live life to the fullest

- "Old": "Still continue to have a busy schedule and social life"

\section{Comparison}

- Young: "More responsible and focused than my peers"

- Middle Age: "Feel I do not seem as old as the people around me who are similarly aged"

- "Old": My health is very good compared to the other women in my family"

Work
- Young: "Feels like I
have gotten a lot
more experience for
a longer time at my
job"
- Middle Age: "My
profession"
"Old": "Actively
engaged in my job"

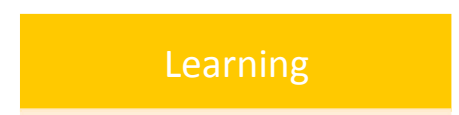

- Young: "Because I am still learning from those who are older than me, as if I'm still in college learning from older professors"

- Middle Age: "No interest in new tech"

- "Old": I’m still learning!"

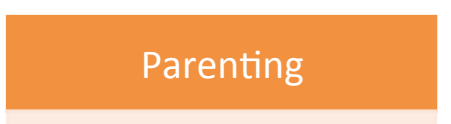

- Young: "I am a single mom"

- Middle Age: "Being a mom"

- "Old": Have 4 children = keeps me young"

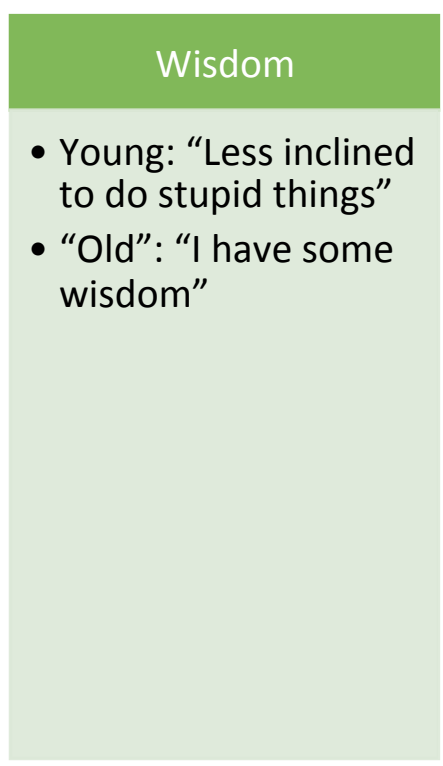


Responsibility

( $n=90)$

Health

(n= 61)

Age Associate

With $(n=57)$

Life Outlook

$(n=53)$

Comparison

$(n=33)$

Work

$(n=21)$

Learning

( $n=14$ )

Parenting

$(n=10)$

Wisdom

$(n=8)$
$44.4 \%$

1 category

$44.3 \%$

1 category

$45.6 \%$

1 category

$43.4 \%$

1 category

$3.0 \%$

1 category

$23.8 \%$

1 category

$7.1 \%$

1 category

$30.0 \%$

1 category

$12.5 \%$

1 category
$42.2 \%$

2 categories

$44.3 \%$

2 categories

$36.8 \%$

2 categories

$37.7 \%$

2 categories

$81.8 \%$

2 categories

$52.4 \%$

2 categories

$64.3 \%$

2 categories

$40.0 \%$

2 categories

$25.0 \%$

2 categories
$12.2 \%$

3 categories

$8.2 \%$

3 categories

$10.5 \%$

3 categories

$13.2 \%$

3 categories

$12.1 \%$

3 categories

$14.3 \%$

3 categories

$21.4 \%$

3 categories

$30.0 \%$

3 categories

WSH 2017, Minneapolis, MN
$1.1 \%$

4 categories

$3.3 \%$

4 categories

$7.0 \%$

4 categories

$5.7 \%$

4 categories

$3.0 \%$

4 categories

$9.5 \%$

4 categories

$7.1 \%$

4 categories

$12.5 \%$

4 categories 


\section{Percentage associated with multiple categories}

\begin{tabular}{|c|c|c|c|c|c|c|c|c|c|}
\hline & Responsibility & Health & Age Assoc With & Life Outlook & Comparison & Work & Learning & Parenting & Wisdom \\
\hline $\begin{array}{l}\text { Responsibility } \\
(n=90)\end{array}$ & $100 \%$ & $3.3 \%$ & $12.2 \%$ & $8.9 \%$ & $28.9 \%$ & $11.1 \%$ & $0 \%$ & $4.4 \%$ & $5.6 \%$ \\
\hline $\begin{array}{l}\text { Health } \\
(n=61)\end{array}$ & $3.3 \%$ & $100 \%$ & $18.0 \%$ & $24.6 \%$ & $6.6 \%$ & $8.2 \%$ & $8.2 \%$ & $1.6 \%$ & $0.0 \%$ \\
\hline $\begin{array}{l}\text { Age Assoc With } \\
(n=57)\end{array}$ & $12.3 \%$ & $15.8 \%$ & $100 \%$ & $14.0 \%$ & 15.8 & 5.3 & 10.5 & 1.8 & 3.5 \\
\hline $\begin{array}{l}\text { Life Outlook } \\
(n=53)\end{array}$ & $13.2 \%$ & $30.2 \%$ & $13.2 \%$ & $100 \%$ & $5.7 \%$ & $7.5 \%$ & $5.7 \%$ & $1.9 \%$ & $3.8 \%$ \\
\hline $\begin{array}{l}\text { Comparison } \\
(n=33)\end{array}$ & $72.7 \%$ & $6.1 \%$ & $27.3 \%$ & $6.1 \%$ & $100 \%$ & $0 \%$ & $0 \%$ & $0 \%$ & $3.0 \%$ \\
\hline $\begin{array}{l}\text { Work } \\
(n=21)\end{array}$ & $52.4 \%$ & $19.0 \%$ & $9.5 \%$ & $14.3 \%$ & $0 \%$ & $100 \%$ & $0 \%$ & $14.3 \%$ & $0 \%$ \\
\hline $\begin{array}{l}\text { Learning } \\
(n=14)\end{array}$ & $0 \%$ & $50.0 \%$ & $42.9 \%$ & $28.6 \%$ & $0 \%$ & $0 \%$ & $100 \%$ & $0 \%$ & $7.1 \%$ \\
\hline $\begin{array}{l}\text { Parenting } \\
(n=10)\end{array}$ & $50.0 \%$ & $10.0 \%$ & $10.0 \%$ & $10.0 \%$ & $0 \%$ & $20.0 \%$ & $0 \%$ & $100 \%$ & $0 \%$ \\
\hline $\begin{array}{l}\text { Wisdom } \\
(n=8)\end{array}$ & $50.0 \%$ & $12.5 \%$ & $25 \%$ & $25 \%$ & 37.5 & $0 \%$ & $12.5 \%$ & $0 \%$ & $100 \%$ \\
\hline
\end{tabular}




\section{Which age groups used each category?}

\begin{tabular}{|l|c|c|c|c|}
\hline Category & $\begin{array}{c}\text { Total } \\
\mathrm{N}=224\end{array}$ & $\begin{array}{c}\text { Young } \\
\mathrm{N}=100\end{array}$ & $\begin{array}{c}\text { Middle-Age } \\
\mathrm{N}=41\end{array}$ & $\begin{array}{c}\text { "Old" } \\
\mathrm{N}=83\end{array}$ \\
\hline Responsibility & $89(39.7 \%)$ & $72(72.0 \%)$ & $13(31.7 \%)$ & $4(4.8 \%)$ \\
\hline Health & $61(27.2 \%)$ & $3(3.0 \%)$ & $6(14.6 \%)$ & $52(62.7 \%)$ \\
\hline Age Assoc & $57(25.4 \%)$ & $22(22.0 \%)$ & $14(34.1 \%)$ & $21(25.3 \%)$ \\
\hline With & $53(23.2 \%)$ & $11(11.0 \%)$ & $9(22.0 \%)$ & $33(39.8 \%)$ \\
\hline Life Outlook & $33(14.7 \%)$ & $23(23.0 \%)$ & $7(17.1 \%)$ & $3(3.6 \%)$ \\
\hline Comparison & $21(9.4 \%)$ & $7(7.0 \%)$ & $8(19.5 \%)$ & $6(7.2 \%)$ \\
\hline Work & $14(6.3)$ & $2(2.0 \%)$ & $2(4.9 \%)$ & $10(12.0 \%)$ \\
\hline Learning & $10(4.5 \%)$ & $1(1.0 \%)$ & $6(14.6 \%)$ & $3(3.6 \%)$ \\
\hline Parenting & $8(3.6 \%)$ & $7(7.0 \%)$ & 0 & $1(1.2 \%)$ \\
\hline Wisdom & & & & \\
\hline
\end{tabular}

